\title{
THE HNN AND GENERALIZED FREE PRODUCT STRUCTURE OF CERTAIN LINEAR GROUPS
}

\author{
BY BENJAMIN FINE \\ Communicated by Hyman Bass, October 15, 1974
}

Introduction. If $d$ is a positive square-free integer let $I_{d}$ be the ring of integers in $Q(\sqrt{ }-d) . I_{d}$ is a Euclidean domain if $d=1,2,3,7,11$. The groups $\operatorname{PSL}_{2}\left(I_{d}\right)=\Gamma_{d}$ over these Euclidean rings have recently been investigated. Methods for generating presentations as well as actual presentations were given in [2], [3] and [8], while these groups were shown to be describable in terms of generalized free products in [3]. Here we announce several extensions of these results suggested by Karrass and Solitar. We show that the Picard group $\Gamma_{1}$ is decomposable directly as a free product with amalgamated subgroup while the groups $\Gamma_{2}, \Gamma_{7}, \Gamma_{11}$ are HNN groups in the sense of [5]. The extensions will be used in [4] to show that these groups are SQ-universal.

Throughout we let $\Gamma_{d}=\operatorname{PSL}_{2}\left(I_{d}\right)$.

The cases $d=1,3$. In [9] it was shown that the Picard group $\Gamma_{1}$ contains a subgroup of finite index which is a generalized free product, while in [3] , $\Gamma_{1}$ was decomposed as a semidirect product with the subgroup above contained as a subgroup of finite index in the normal factor. Also, in [3], $\Gamma_{3}$ was shown to have a subgroup of finite index which is again a free product with amalgamated subgroup. As a strengthening of these we obtain

THEOREM. The Picard group $\Gamma_{1}$ is given directly as a free product of 2 groups $G_{1}, G_{2}$ with amalgamated subgroup $H . G_{1}$ is the free product of the symmetric group $\Sigma_{3}$ and an alternating group $A_{4}$ with a 3-cycle amalgamated; while $G_{2}$ is $D$ with a 2-cycle amalgamated. The amalgamated subgroup $H \simeq \operatorname{PSL}_{2}(Z)$ (modular group).

Proof. The result follows directly from a presentation of the Picard group $\Gamma_{1}$, given by $G$. Sansone [7]:

AMS (MOS) subject classifications (1970). Primary 20G20, 20E30; Secondary $20 \mathrm{E} 35$.

Key words and phrases. HNN group, generalized free product, free product with amalgamation, SQ-universal.

Copyright $\odot$ 1975, American Mathematical Society 


$$
\begin{aligned}
\Gamma_{1} \simeq & \left\{A, B, C, D ; A^{3}=B^{2}=C^{3}=D^{2}\right. \\
& \left.=(A C)^{2}=(A D)^{2}=(B C)^{2}=(B D)^{2}=1\right\} .
\end{aligned}
$$

A different decomposition of $\Gamma_{1}$ as a free product with amalgamation was computed by Karrass and Solitar in [6]. This was used to investigate the nilpotent subgroups of $\Gamma_{1}$.

A similar statement cannot be made for $\Gamma_{3}$. In fact

THEOREM (KARRASS AND SOlitAR [6]). The group $\Gamma_{3}$ is indecomposable as a generalized free product.

The proof depends on a technical lemma involving elements of a finite order in a generalized free product.

HNN groups; cases $d=2,7,11$. A group of the form

$$
G=\left\{t_{1} \cdots t_{n}, K, \text { rel } K, t_{i} L_{i} t_{i}^{-1}=\phi\left(L_{i}\right)\right\}
$$

where $K$ is a group, $L_{i}$ are subgroups of $K \phi_{i}$, a collection is isomorphisms $L_{i} \longrightarrow \phi\left(L_{i}\right)$ is called an HNN group [5]. $K$ is called the base, $\left(L_{i}, \phi\left(L_{i}\right)\right)$ associated subgroups and the group generated by $t_{1}, \cdots, t_{n}$, the free part.

A subgroup structure theory for HNN groups paralleling that of free products with amalgamations has been developed [1], [5]. Here we state that the groups $\Gamma_{2}, \Gamma_{7}, \Gamma_{11}$ are HNN groups with free parts of rank 1 and bases which are generalized free products while $\Gamma_{1}, \Gamma_{3}$ are indecomposable as HNN groups.

First we obtain the following

LEMmA. The groups $\Gamma_{1}, \Gamma_{3}$ are not HNN groups.

An HNN group must have a free quotient so this follows directly from the even stronger statement:

LEMmA. $\Gamma_{1}, \Gamma_{3}$ have no torsion free quotients.

Proof. In [3] we showed that $\Gamma_{1}, \Gamma_{3}$ are generated by elements of finite order (either 2 or 3 ). Therefore in any torsion free quotient these must map on the identity.

Of the remaining 3 groups we can state

THEOREM. The groups $\Gamma_{2}, \Gamma_{7}, \Gamma_{11}$ are $H N N$ groups with bases $K_{2}, K_{7}$, $K_{11}$ and free parts of rank 1. Further in each case the base group is a free product with amalgamated subgroups. In each case the associated subgroups 
are modular groups $\simeq \operatorname{PSL}_{2}(Z)$ while the factors of the bases are particularly accessible (free groups, free abelian groups, finite Fuchsian groups or free products of these); explicitly

(i) $K_{2}$ is $G_{1} * G_{2}$ with $H$ amalgamated; $G_{1} \simeq Z_{2} \times Z_{2}, G_{2} \simeq A_{4} \simeq$ $\operatorname{PSL}(2,3)$ and $H \simeq Z_{2}$.

(ii) $K_{7}$ is $G_{1} * G_{2}$ with $H$ amalgamated where $G_{1} \simeq \Sigma_{3}, G_{2} \simeq \Sigma_{3}$ and $H \simeq Z_{2}$.

(iii) $K_{11}$ is $G_{1} * G_{2}$ with $H$ amalgamated; $G_{1} \simeq A_{4}, G_{2} \simeq A_{4}$ and $H \simeq Z_{3}$.

The proofs follow from detailed investigations of the presentations of these groups developed in [3] and [8]. The decomposition of these groups as HNN groups with bases as generalized free products are used in [4] to investigate the SQ-universality of these groups.

The non-Euclidean cases. Analogous results are true for those $\Gamma_{d}$ whose presentations have been computed over non-Euclidean rings $I_{d}, d \neq 1,2,3$, 7,11 . These are HNN groups with free parts of rank 1, and bases which are generalized free products. However the factors and the general structure of the base becomes increasingly complex. As an example we state

THEOREM. The group $\Gamma_{5}=\operatorname{PSL}_{2}\left(I_{5}\right)$ is an HNN group with free part of rank 1 and base $K_{5}$. Further $K_{5}$ is the free product of 2 groups $G_{1}, G_{2}$ with subgroup $H$ amalgamated;

$G_{1} \simeq$ free product of the modular group $\operatorname{PSL}_{2}(Z)$ and $Z \times Z_{3}$ with a 3-cycle amalgamated.

$G_{2} \simeq$ free product of $Z_{2} \times Z_{2} \times Z_{2}$ and $Z \times Z_{2}$ with a 2-cycle amalgamated.

$$
H \simeq Z \times Z_{2} .
$$

The single pair of associated subgroups in $K_{5}, N, M$ are $\simeq Z \times Z_{2}$.

The proof follows as in the Euclidean cases by examining the presentation $\Gamma_{5}$ given in [8]. We also note that $\Gamma_{5}$ as well as the other groups over the non-Euclidean rings with class number $>1$, whose presentations were computed by Swan in [8], are also HNN groups with free parts of rank 2, and slightly simpler bases. HNN groups with free parts of rank $>1$ are SQ-universal [4]. A thorough investigation of the non-Euclidean cases will be done elsewhere. 


\section{REFERENCES}

1. R. G. Burns, Finitely generated subgroups of HNN groups, Canad. J. Math. 25 (1973), 1103-1112.

2. P. M. Cohn, $A$ presentation of $\mathrm{SL}_{2}$ for Euclidean imaginary quadratic number fields, Mathematika 15 (1968), 156-163. MR 38 \#4568.

3. Benjamin Fine, The structure of $\mathrm{PSL}_{2}(R)$, Ann. of Math. Studies, no. 79, Discontinuous groups and Riemann surfaces, May 1973.

4. Benjamin Fine and Marvin Tretkoff, The SQ-universality of certain linear groups (to appear).

5. A. Karrass and D. Solitar, Subgroups of HNN groups and groups with one defining relation, Canad. J. Math. 23 (1971), 627-643. MR 46 \#260.

6. —, (to appear).

7. G. Sansone, I sotiogruppe del gruppo di Picard e due teoremi sui gruppi finiti analoghi at teorema del dyck. Rend. Circ. Mat. Palermo 47 (1923), 273.

8. R. G. Swan, Generators and relations for certain special linear groups, Advances in Math. 6 (1971), 1-77.

9. H. V. Waldinger, On the subgroups of the Picard group, Proc. Amer. Math. Soc. 16 (1965), 1373-1378. MR 32 \#647.

DEPARTMENT OF MATHEMATICS, FAIRFIELD UNIVERSITY, FAIRFIELD, CONNECTICUT 06430 